Pacific Journal of Mathematics

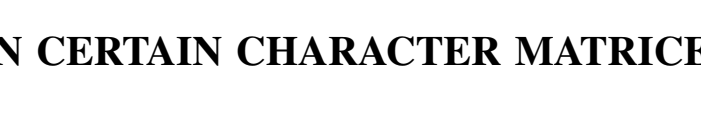




\section{ON CERTAIN CHARACTER MATRICES}

\section{H. Lehmer}

For only a very limited class of matrices $M$ is it possible to give explicit formulas for the determinant, characteristic roots and inverse of $M$ as well as the general element of $M^{k}$. Nontrivial instances of such sets of matrices are useful as examples in testing the correctness and efficacy of various matrix computing routines especially when the elements are small integers or simple rational numbers. The purpose of this paper is to indicate two new classes of such matrices which arise from the theory of exponential sums and have as general elements simple functions involving real nonprinciple characters or Legendre symbols.

The same method of determining characteristic roots is used for both types of matrices. It depends on the fact that the roots of a polynomial are determined by the sums of their like powers. That is, if $S_{k}$ denotes the sum of the $k$ th powers of the roots of a polynomial of degree $n$ and if complex numbers $\rho_{1}, \rho_{2}, \cdots, \rho_{n}$ are exhibited for which

$$
\sum_{i=1}^{n} \rho_{i}^{k}=S_{k} \quad(k=1, \cdots, n),
$$

then the $\rho_{i}$ are the roots of the polynomial.

All matrices $M$ are square and of order $p-1$ where $p$ is an odd prime. Their elements involve Legendre's symbol $\chi(n)$ defined by

$$
\chi(n)=\left(\begin{array}{c}
n \\
p
\end{array}\right)=\left\{\begin{aligned}
0 & \text { if } p \text { divides } n \\
-1 & \text { if the congruence } x^{2} \equiv n(\bmod p) \text { is impossible } \\
+1 & \text { otherwise }
\end{aligned}\right.
$$

Thus for $p=7$

$$
\begin{array}{lll}
\chi(0)=0 & \chi(1)=1 & \chi(2)=1 \quad \chi(3)=-1 \\
\chi(4)=1 & \chi(5)=-1 & \chi(6)=-1 .
\end{array}
$$

Besides the simple properties

$$
\begin{aligned}
& \chi(i) \chi(j)=\chi(i j) \\
& \chi(i+p)=\chi(i)
\end{aligned}
$$

Received August 26, 1955. A part of the results of this paper were obtained in 1952 under a contract between the Offlce of Naval Research and the National Bureau of Standards. 
we use the following identities

$$
\begin{gathered}
\sum_{k=1}^{p-1} \chi(n+k)=-\chi(n) \\
\sum_{k=1}^{p-1} \chi(m+k) \chi(n+k)=\delta_{m}^{n}-\chi(m) \chi(n)-1
\end{gathered}
$$

where $\delta_{m}^{n}$ is Kronecker's delta $\bmod p$, that is

$$
\delta_{a}^{b}=\left\{\begin{array}{l}
1 \text { if } a \equiv b(\bmod p) \\
0 \text { otherwise . }
\end{array}\right.
$$

Identity (1) states the familiar fact that there are as many quadratic residues as non-residues of $p$.

Identity (2) is apparently due to Jacobsthal [1] and a simple proof is given in $\S 4$.

2. Matrices of the first kind. Let $a, b, c, d$ be any four numbers. We consider the matrix $M=M(a, b, c, d)$ whose general element $a_{i 1}$ is

$$
a_{i j}=a+b \chi(i)+c \chi(j)+d \chi(i j)
$$

and denote the general element of $M^{k}$ by $a_{i j}^{(k)}$.

Theorem 1. The general element of $M^{k}(a, b, c, d)$ is

$$
a_{i j}^{(k)}=(p-1)^{k-1}\left\{a_{k}+b_{k} \chi(i)+c_{k} \chi(j)+d_{k} \chi(i j)\right\}
$$

where $k$ is a positive integer and

$$
\left(\begin{array}{cc}
a_{k} & b_{k} \\
c_{k} & d_{k}
\end{array}\right)=\left(\begin{array}{ll}
a & b \\
c & d
\end{array}\right)^{k}
$$

Proof. The assertion of the theorem is trivial for the case $k=1$. If true for $k=n$, we have

$$
\begin{aligned}
a_{i j}^{(n+1)} & =\sum_{r=1}^{p-1} a_{i r} a_{r j}^{(n)} \\
& =\sum_{r=1}^{p-1}\{a+b \chi(i)+c \chi(r)+d \chi(i r)\}\left\{a_{n}+b_{n} \chi(r)+c_{n} \chi(j)+d_{n} \chi(r j)\right\} .
\end{aligned}
$$

Multiplying together the two factors under summation and using the facts that

$$
\chi^{2}(r)=1, \quad \sum_{r=1}^{p-1} \chi(r)=0
$$


we find

$$
a_{i j}^{(n+1)}=(p-1)[A+B \chi(i)+C \chi(j)+D \chi(i j)],
$$

where

$$
\begin{array}{ll}
A=a a_{n}+c b_{n} & B=b a_{n}+d b_{n} \\
C=a c_{n}+c d_{n} & D=b c_{n}+d d_{n}
\end{array}
$$

Hence

$$
\left(\begin{array}{ll}
A & B \\
C & D
\end{array}\right)=\left(\begin{array}{ll}
a_{n} & b_{n} \\
c_{n} & d_{n}
\end{array}\right)\left(\begin{array}{ll}
a & b \\
c & d
\end{array}\right)=\left(\begin{array}{ll}
a & b \\
c & d
\end{array}\right)^{n+1},
$$

Thus the induction from $n$ to $n+1$ is completed.

THEOREM 2. The characteristic roots of $M(a, b, c, d)$ are

$$
(p-1) \rho_{1},(p-1) \rho_{2}, 0,0, \cdots, 0
$$

where $\rho_{1}, \rho_{2}$ are the characteristic roots of the matrix

$$
N=\left(\begin{array}{ll}
a & b \\
c & d
\end{array}\right)
$$

that is, $\rho_{1}, \rho_{2}$ are the roots of the equation

$$
\lambda^{2}-(a+b) \lambda+a d-b c=0 \text {. }
$$

Proof. Let $k$ be any positive integer, and denote by $\sigma_{k}$ the sum of the $k$ th powers of the characteristic roots of $M(a, b, c, d)$. Since $\sigma_{k}$ is thus the trace of $M^{k}$ we have by Theorem 1

$$
\sigma_{k}=\sum_{i=1}^{p-1} a_{i i}^{(k)}=(p-1)^{k-1} \sum_{i=1}^{p-1}\left\{a_{k}+b_{k} \chi(i)+c_{k} \chi(i)+d_{k} \chi\left(i^{2}\right)\right\}=(p-1)^{k}\left(a_{k}+d_{k}\right)
$$

by (3).

Now $a_{k}+d_{k}$, being the trace of $N^{k}$, has the value $\rho_{1}^{k}+\rho_{2}^{k}$ where $\rho_{1}, \rho_{2}$ are the characteristic roots of $N$.

Therefore

$$
\sigma_{k}=\left\{(p-1) \rho_{1}\right\}^{k}+\left\{(p-1) \rho_{2}\right\}^{k}+0^{k}+\cdots+0^{k} .
$$

Since this holds for all positive integers $k$ and since the roots of a polynomial are determined by their sums of powers, it follows that the characteristic roots of $M$ are those specified in the conclusion of the theorem.

Except for the case $p=3$ the matrix $M(a, b, c, d)$ is singular and 
so has no inverse. For $p=3$ the characteristic roots being, $2 \rho_{1}, 2 \rho_{2}$ the determinant of $M$ is $4 \rho_{1} \rho_{2}=4(a d-b c)$ and its inverse (assuming that $a d-b c \neq 0)$ is given by

$$
4(a d-b c) M^{-1}=\left(\begin{array}{rr}
a-b-c+d & -a-b+c+d \\
-a+b-c+d & a+b+c+d
\end{array}\right) .
$$

The rank of $M$ which is in general 2 will become 1 if and only if $a d-b c=0$; that is, if and only if the general term is a product of two factors

$$
a_{i i}=\{x+y \chi(i)\}\{z+w \chi(j)\}
$$

3. Matrices of the second kind. Let $c$ be any constant and $\alpha$ an integer. We define $A_{p}=A_{p}(c, \alpha)$ as the matrix whose general element is

$$
a_{i j}=c+\chi(\alpha+i+j) \text {. }
$$

The properties of this matrix are more recondite than those of $M(a, b$, $c, d)$. The general element $\alpha_{i j}^{(2)}$ of $A_{p}^{2}$ is not of the same form as $a_{i j}$ but is

$$
\begin{aligned}
a_{i j}^{(2)} & =\sum_{r=1}^{p-1}\{c+\chi(\alpha+i+r)\}\{c+\chi(\alpha+r+j)\} \\
& =(p-1) c^{2}-1-c[\chi(\alpha+i)+\chi(\alpha+j)]-\chi(\alpha+i) \chi(\alpha+j)+p \delta_{i}^{j}
\end{aligned}
$$

as we see by applying (2). This prompts us to define a function $\psi_{k}(i, j)$ and three sequences $C_{k}, S_{k}$ and $P_{k}$ by

$$
\begin{gathered}
\psi_{k}(i, j)= \begin{cases}p^{k / 2} \delta_{i}^{j} & \text { if } k \text { is even } \\
p^{(k-1) / 2} \chi(\alpha+i+j) & \text { if } k \text { is odd }\end{cases} \\
a_{i j}^{(k)}=C_{k}+S_{k}[\chi(\alpha+i)+\chi(\alpha+j)]+P_{k} \chi(\alpha+i) \chi(\alpha+j)+\psi_{k}(i, j)
\end{gathered}
$$

so that initially

$$
\begin{array}{lll}
C_{0}=0 & C_{1}=c & C_{2}=(p-1) c^{2}-1 \\
S_{0}=0 & S_{1}=0 & S_{2}=-c \\
P_{0}=0 & P_{1}=0 & P_{2}=-1 .
\end{array}
$$

If we substitute from (4) and (5) into the relation

$$
a_{i j}^{(k+1)}=\sum_{r=1}^{p-1} a_{i r} a_{r j}^{(k)}
$$

we are led to a system of difference equations to determine $C_{k}, S_{k}$ and $P_{k}$. Because of the nature of the function $\psi$ these depend upon the 
parity of $k$. Setting $k=2 m$ and $2 m+1$ in turn we find

$$
\begin{aligned}
& C_{2 m+1}=(p-1) c C_{2 m}-[1+c \chi(\alpha)] S_{2 m}+c p^{m} \\
& S_{2 m+1}=(p-1) c S_{2 m}-[1+c \chi(\alpha)] P_{2 m}=-\chi(\alpha) S_{2 m}-C_{2 m} \\
& P_{2 m+1}=-S_{2 m}-\chi(\alpha) P_{2 m}
\end{aligned}
$$

and

$$
\begin{aligned}
C_{2 m+2} & =(p-1) c C_{2 m+1}-[1+c \chi(\alpha)] S_{2 m+1}-p^{m} \\
S_{2 m+2} & =(p-1) c S_{2 m+1}-[1+c \chi(\alpha)] P_{2 m+1}-c p^{m} \\
& =-\chi(\alpha) S_{2 m+1}-C_{2 m+1} \\
P_{2 m+2} & =-S_{2 m+1}-\chi(\alpha) P_{2 m+1}-p^{m} .
\end{aligned}
$$

For simplicity in what follows we write $\chi$ for $\chi(\alpha)$. By simple elimination the above equations may be replaced by second order ones in which the variables are separated as follows.

$$
\begin{aligned}
& C_{2 m+1}=A C_{2 m}+B C_{2 m-1}+(c p-\chi) p^{m-1} \\
& C_{2 m+2}=A C_{2 m+1}+B C_{2 m}+(c \chi-1) p^{m} \\
& S_{2 m+1}=A S_{2 m}+B S_{2 m-1}+p^{m-1} \\
& S_{2 m+2}=A S_{2 m+1}+B S_{2 m}-c p^{m} \\
& P_{2 m+1}=A P_{2 m}+B P_{2 m-1}+c p^{m} \\
& P_{2 m+2}=A P_{2 m+1}+B P_{2 m}-p^{m}
\end{aligned}
$$

where we have written

$$
c(p-1)-\chi=A \quad c p \chi+1=B .
$$

We define a sequence $W_{k}$ by

$$
W_{k}=(p-1) C_{k}-2 \chi S_{k}+\left(p-1-\chi^{2}\right) P_{k}
$$

so that

$$
W_{0}=0, \quad W_{1}=c(p-1),
$$

and a sequence $T_{k}$ by

$$
\begin{aligned}
& T_{2 m+1}=W_{2 m+1}-\chi p^{m} \\
& T_{: 2 m}=W_{: m}+(p-1) p^{m}
\end{aligned}
$$

with initial values 


$$
T_{0}=p-1, \quad T_{1}=A
$$

From (7)-(12) we deduce

$$
\begin{gathered}
T_{2 m+1}=A T_{2 m}+B T_{2 m-1}-A P^{m}(p-3) \\
T_{2 m+2}=A T_{2 m+1}+B T_{2 m}+(p-B) p^{m}(p-3) .
\end{gathered}
$$

This suggests a final substitution:

$$
V_{k}= \begin{cases}T_{k}-(p-3) p^{k / 2} & \text { if } k \text { is even } \\ T_{k} & \text { if } k \text { is odd }\end{cases}
$$

in terms of which (15) becomes simply

$$
V_{k+1}=A V_{k}+B V_{k-1}
$$

with

$$
V_{0}=2, \quad V_{1}=A \text {. }
$$

We are now in a position to prove the following.

THEOREM 3. The characteristic roots of the matrix

$$
A_{p}=\left(a_{i j}\right)=(c+\chi(\alpha+i+j))
$$

consist in $p^{1 / 2}$ and $-p^{1 / 2}$ each occurring with multiplicities $(p-3) / 2$ and, in addition, the two roots of the quadratic equation

$$
\lambda^{2}-[c(p-1)-\chi(\alpha)] \lambda-c p \chi(\alpha)-1=0 .
$$

Using the abbreviations (13) we may restate the theorem by asserting that the characteristic equation of $A_{p}$ is

$$
\left(\lambda^{2}-p\right)^{(p-3) / 2}\left(\lambda^{2}-A \lambda-B\right)=0 \text {. }
$$

Proof. We begin by noting that, from the definition (4),

$$
\sum_{i=1}^{p-1} \psi_{k}(i, i)= \begin{cases}(p-1) p^{k / 2} & \text { if } k \text { is even } \\ -\chi(\alpha) p^{(k-1) / 2} & \text { if } k \text { is odd } \\ =T_{k}-W_{k} . & \end{cases}
$$

Hence if we set $i=j$ in (5) and sum over $i$ we obtain

$$
\sum_{i=1}^{p-1} a_{i i}^{(k)}=(p-1) C_{k}-2 \chi S_{k}+\left(p-1-\chi^{2}\right) P_{k}+T_{k}-W_{k}=T_{k}
$$

That is to say, the function $T_{k}$ defined previously by (14) is the trace of the $k$ th power of our matrix $A_{p}$. 
Turning now to the function $V_{k}$, and denoting the two roots of $\lambda^{2}-A \lambda-B=0$ by $\rho_{1}$ and $\rho_{2}$ we see that

$$
V_{0}=\rho_{1}^{0}+\rho_{2}^{0}, \quad V_{1}=\rho_{1}+\rho_{2}
$$

and in general by induction from $k$ and $k-1$ to $k+1$

$$
V_{k+1}=A V_{k}+B V_{k-1}=\left(\rho_{1}+\rho_{2}\right)\left(\rho_{1}^{k}+\rho_{2}^{k}\right)-\rho_{1} \rho_{2}\left(\rho_{1}^{k-1}+\rho_{2}^{k-1}\right)=\rho_{1}^{k+1}+\rho_{2}^{k+1} .
$$

Therefore (16) can be written in the form

$$
T_{k}=\rho_{1}^{k}+\rho_{2}^{k}+\frac{p-3}{2}\left(p^{1 / 2}\right)^{k}+\frac{p-3}{2}\left(-p^{1 / 2}\right)^{k} .
$$

Since $T_{k}$ is the trace of $A_{j}^{k}$ and so is the sum of $k$ th powers of characteristic roots of $A_{p}$, it follows from (17) that these roots must be $\rho_{1}, \rho_{2}$ and $\pm p^{1 / 2}$ the latter two having multiplicities $(p-3) / 2$.

As a corollary to Theorem 3 we obtain the determinant of $A_{p}$ as the product of its characteristic roots, namely

$$
\rho_{1} \rho_{2}(-p)^{(p-3) / 2}=\chi(-1) B p^{(p-3) / 2}=\chi(-1)[1+c p \chi(\alpha)] p^{(p-3) / 2} .
$$

It follows that $A_{p}$ is nonsingular provided $c$ is not chosen as the negative reciprocal of $p \chi(\alpha)$, in other words provided $B \neq 0$.

The inverse of $A_{p}$ is easily obtained. We simply substitute $m=0$ into (7), (9) and (11) and use the initial conditions (6) to find (assuming $B \neq 0$ ),

$$
\begin{aligned}
& C_{-1}=\chi(\alpha) /(p B) \\
& S_{-1}=-1 /(p B) \\
& P_{-1}=-c / B
\end{aligned}
$$

and

$$
\psi_{-1}(i j)=\chi(\alpha+i+j) / p .
$$

Hence for the general element $a_{i j}^{-1}$ of $A_{p}^{-1}$ we find

$$
p B a_{i j}^{-1}=\chi(\alpha)-\chi(\alpha+i)-\chi(\alpha+j)-c p \chi(\alpha+i) \chi(\alpha+j)+B \chi(\alpha+i+j) .
$$

The reader may wish to verify, as an exercise in the use of (1) and (2), that (18) is indeed correct.

The general element $a_{i j}^{(k)}$ of $A_{p}^{k}$ for an arbitrary integer $: k$ can be found in the form (5) by solving the difference equations (7)-(12) for $C_{k}, S_{k}$, and $P_{k}$ as we did for the special combination we denoted by $T_{k}$. These functions are linear combinations of the $k$ th powers of the characteristic roots of $A_{p}$. Various special cases are sufficiently simple 
to be interesting and useful. The cases of $c=0$ involve in general the Fibonacci numbers. For $c=0$ and $\alpha=0$ the reader will find that

$$
\begin{gathered}
a_{i j}^{(2 m)}=p^{m} \delta_{i}^{j}-\frac{p^{m}-1}{p-1}\{\chi(i j)+1\} \\
a_{i j}^{(2 m+1)}=p^{m} \chi(i+j)+\frac{p^{m}-1}{p-1}\{\chi(i)+\chi(j)\} .
\end{gathered}
$$

Thus the inverse of the matrix

$$
a_{i j}=\chi(i+j)
$$

has for its general element

$$
a_{i j}^{-1}=[\chi(i+j)-\chi(i)-\chi(j)] / p
$$

which comes from putting $m=-1$ into (19).

4. Proof of Jacobsthal's Identity. To prove (2) we may write

$$
S(a, b)=\sum_{k=0}^{p-1} \chi(a+k) \chi(b+k) .
$$

Substituting $r$ for $a+k$ and using the periodicity of $\chi$ we obtain

$$
S(a, b)=S(0, b-a),
$$

so that $S(a, b)$ depends only on the difference between its variables. If this difference is zero we have

$$
S(0,0)=\sum_{k=0}^{p-1} \chi^{2}(k)=p-1 .
$$

If the difference $b-a=\delta \neq 0$, we replace $k$ by $t \hat{o}(\bmod p)$ and write

$$
S(0, \delta)=\sum_{k=0}^{p-1} \chi(k) \chi(\delta+k)=\sum_{t=0}^{p-1} \chi(t \delta) \chi(\delta+t \delta)=\chi^{2}(\delta) \sum_{t=0}^{p-1} \chi(t) \chi(t+1)=S(0,1) .
$$

Thus $S(0, \delta)$ is not a function of $\delta$. That is

$$
(p-1) S(0,1)=\sum_{\delta=1}^{p-1} S(0, \delta)=\sum_{k=0}^{p-1} \chi(k) \sum_{\delta=1}^{p-1} \chi(k+\delta)=-\sum_{k=0}^{p-1} \chi^{2}(k)=-(p-1) .
$$

Hence $S(0, \delta)=-1$ if $\delta \neq 0$. Thus in general we have

$$
S(a, b)=p \delta_{a}^{b}-1 \text {. }
$$

From this (2) follows at once.

The referee has called my attention to the fact that certain matrices of order $p+1=4 k$ involving $\chi(i-j)$ have been considered by Payley [2] 
and Gilman from different point of view. Their results depend also on Jacobsthal's identity.

\section{REFERENCES}

1. E. Jacobsthal, Andwendungen einer Formel aus der Theorie der quadratischen Restc, Dissertation, Berlin 1906.

2. R. E. A. C. Payley, On orthogonal matrices, Jour. Math. Phys. 12 (1933), 311-320.

UNIVERSITY OF CALIFORNIA, BERKELEY 



\section{PACIFIC JOURNAL OF MATHEMATICS}

\section{EDITORS}

\author{
H. L. Royden \\ Stanford University \\ Stanford, California \\ E. Hewitt \\ University of Washington \\ Seattle 5 , Washington
}

R. P. Dilworth

California Institute of Technology

Pasadena 4, California

\author{
A. HorN* \\ University of California \\ Los Angeles 24, California
}

\section{ASSOCIATE EDITORS}

\author{
E. F. BECKENBACH \\ C. E. BURGESS \\ H. BUSEMANN \\ H. FEDERER
}

\author{
M. HALL \\ P. R. HALMOS \\ V. GANAPATHY IYER \\ R. D. JAMES
}

\author{
M. S. KNEBELMAN \\ I. NIVEN \\ T. G. OSTROM \\ M. M. SCHIFFER
}

J. J. STOKER

G. SZEKERES

F. WOLF

K. YOSIDA

\section{SPONSORS}

\author{
UNIVERSITY OF BRITISH COLUMBIA \\ CALIFORNIA INSTITUTE OF TECHNOLOGY \\ UNIVERSITY OF CALIFORNIA, BERKELEY \\ UNIVERSITY OF CALIFORNIA, DAVIS \\ UNIVERSITY OF CALIFORNIA, LOS ANGELES \\ UNIVERSITY OF CALIFORNIA, SANTA BARBARA \\ MONTANA STATE UNIVERSITY \\ UNIVERSITY OF NEVADA \\ OREGON STATE COLLEGE \\ UNIVERSITY OF OREGON \\ UNIVERSITY OF SOUTHERN CALIFORNIA
}

STANFORD RESEARCH INSTITUTE

STANFORD UNIVERSITY

UNIVERSITY OF UTAH

WASHINGTON STATE COLLEGE

UNIVERSITY OF WASHINGTON

AMERICAN MATHEMATICAL SOCIETY HUGHES AIRCRAFT COMPANY SHELL DEVELOPMENT COMPANY

Mathematical papers intended for publication in the Pacific Journal of Mathematics should be typewritten (double spaced), and the author should keep a complete copy. Manuscripts may be sent to any of the editors. Manuscripts intended for the outgoing editors should be sent to their successors. All other communications to the editors should be addressed to the managing editor, Alfred Horn at the University of California, Los Angeles 24, California.

50 reprints of each article are furnished free of charge; additional copies may be obtained at cost in multiples of 50 .

The Pacific Journal of Mathematics is published quarterly, in March, June, September, and December. The price per volume (4 numbers) is $\$ 12.00$; single issues, $\$ 3.50$. Back numbers are available. Special price to individual faculty members of supporting institutions and to individual members of the American Mathematical Society: $\$ 4.00$ per volume; single issues, $\$ 1.25$.

Subscriptions, orders for back numbers, and changes of address should be sent to Pacific Journal of Mathematics, c/o University of California Press, Berkeley 4, California.

Printed at Kokusai Bunken Insatsusha (International Academic Printing Co., Ltd.), No. 10, 1-chome, Fujimi-cho, Chiyoda-ku, Tokyo, Japan.

* During the absence of E. G. Straus.

PUBLISHED BY PACIFIC JOURNAL OF MATHEMATICS, A NON-PROFIT CORPORATION COPYRIGHT 1956 BY PACIFIC JOURNAL OF MATHEMATICS 


\section{Pacific Journal of Mathematics}

\section{Vol. 6, No. $3 \quad$ BadMonth, 1956}

Richard Arens and James Eells, Jr., On embedding uniform and topological spaces ............................................ 397

N. Aronszajn and Prom Panitchpakdi, Extension of uniformly continuous transformations and hyperconvex metric spaces .............. 405

Kai Lai Chung and Cyrus Derman, Non-recurrent random walks ........ 441

Harry Herbert Corson, III, On some special systems of equations . . . . . . . . . 449

Charles W. Curtis, On Lie algebras of algebraic linear transformations . . . 453

Isidore Heller, Neighbor relations on the convex of cyclic permutations . . . . 467

Solomon Leader, Convergence topologies for measures and the existence of transition probabilities..................................... 479

D. H. Lehmer, On certain character matrices ...................... 491

Michael Bahir Maschler, Minimal domains and their Bergman kernel function ........................................... 501

Wm. M. Myers, Functionals associated with a continuous transformation ................................... 517

Irving Reiner and Jonathan Dean Swift, Congruence subgroups of matrix groups ....................................... 529

Andrew Sobczyk, Simple families of lines ................... 541

Charles Standish, A class of measure preserving transformations ........ 553 Jeremiah Milton Stark, On distortion in pseudo-conformal mapping ..... 565 